\title{
Overview of Tidal Energy with its Benefits and Drawbacks
}

\author{
Shivam Dixit \\ M.Tech Scholar \\ Department of Energy Technology \\ Truba Institute of Engineering \& Information \\ Technology \\ Bhopal, M.P, India \\ sdixit5333333@gmail.com
}

\author{
Prof. Pankaj Badgaiyan \\ Assistant Professor \\ Department of Energy Technology \\ Truba Institute Of Engineering \& Information \\ Technology \\ Bhopal, M.P, India
}

\begin{abstract}
The Tidal power is a type of hydropower that captures the power of the tides to generate electricity and perhaps other sources of energy. Tidal power, while not widely been using presently, it has potential to deliver electricity in the upcoming years. Tidal energy is a type of gravitational hydropower that uses flow of water to accelerate a turbine as well as generate energy. Researchers have discussed the various types of tidal energy and also the performance of various researchers in this field in this article.
\end{abstract}

Keywords: Tidal Energy, Tides, Barrages, tidal streams, energy generator, tidal lagoons.

\section{INTRODUCTION}

Wind and solar energy are less predictable than tides. Tidal power has traditionally had a high cost and restricted availability of locations with sufficiently large tidal ranges or flow velocities, limiting its total availability among renewable energy sources. However, many recent technological advancements and improvements, both in design (e.g., dynamic tidal power, tidal lagoons) and turbine technology (e.g., new axial turbines, cross flow turbines), suggest that total tidal energy availability may be much higher than previously assumed, and that economic and environmental costs may be dragged down to realistic prices.

Tides are the periodic motion of the waters of the sea due to the inter-attractive forces between thecelestial bodies. Tides are very long-period waves that move through the oceans in response to theforces exerted by the moon and sun. Tide and current are not the same. Tide is the vertical rise and fall of the water and tidal current is the horizontal flow. In simple words, the tide rises and falls, the tidal current floods and ebbs. The principal of tidal forces are generated by the Moon and Sun. The Moon is the main tide-generating body. Due to its greater distance, the Sun's effect is only 46 per cent of the Moon's.
Tidal power is usually generated by building a dam across a tidal basin's entrance. The dam has a gate that opens to enable the tide to flow into the basin; the gate then closes, and typical hydropower technologies may be utilized to produce electricity from the raised water in the basin when the sea level decreases.

\section{A. Tidal Energy Generator}

Tidal energy is generated by the movement of our tides and seas, where the intensity of the water from tidal rise and fall is a type of kinetic energy. Tidal power is a subset of gravitational hydropower, which uses the flow of water to propel a turbine and create energy. The turbines are similar to wind turbines; however, they are located underwater.

Tidal energy can be obtained using three methods: tidal streams, barrages, tidal lagoons.

The term "tidal stream" refers to the horizontally flow thru the oceans generated by the tide's constant flow \& flooding, which is the vertically up-down motion of the planet's water. A tidal flow or high tide current, with exception of moving water, which are constant, unilateral, and form a stable lateral flow of water streaming down a river or stream, etc., keeps changing its speed, guidance, and lateral motion on a regular basis depending on the powers of the tide attempting to control it.

Unlike tidal wall power, that uses a protective wall to harvest the energy, tidal stream generating is a quasi tidal technique. Tidal current devices are hydrokinetic power sources that capture angular momentum (energy in motion) from flowing water produced by the tide without affecting the environment. The ebb and flow of the tides leads the ocean waters to pile up at or near water, resulting in a greater tide all along shore. Some of this water is pushed into littoral zones, basin, and bays, but the rest is forced laterally all along shore. The tidal range motion, which is magnified by physical characteristics along the coast, concentrates these 
water currents into a single regular and focused kind of renewable power that we can use.

In many aspects, the concepts of tidal current generating are comparable to those of wind energy. Horizontal dynamic engine known as "tidal barrages" or "tidal stream turbines" are located on the ocean bottom, with stream starts moving over the turbines, driving a generating in the same way that wind turns wind turbine blades. However, in some tidal current production locations, the seabed resembles an undersea wind turbine, with vast arrays of tidal current turbines. The produced tidal energy is subsequently transported to the coast via undersea cables, which are lengthy underwater electrical cables. Overseas tidal generators can be partially or completely buried beneath the water's surface, with partially buried turbines proving easier to maintain and less costly for maintenance.

As the kinetic energy content of a tidal stream flows per unit time, which is the same as the hydro power ( $\mathrm{P}$ ), the available energy can be calculated in terms of velocity ( $\mathrm{V}$ ), swept cross-sectional area ( A ) perpendicular to the stream flow direction, and the density of the water $(\rho)$, which for sea water is approximately $1025 \mathrm{~kg} / \mathrm{m} 3$. Providing the velocity is uniform across the cross-sectional area, at any instant in the tidal cycle the amount of energy available will be: $\mathrm{P}=1 / 2 \rho \mathrm{AV}^{3}$.

Tidal energy is more potent than wind energy because water is considerably denser than air. Tides, unlike wind, are predictable and steady. Tidal generators generate a consistent, dependable supply of energy wherever they are utilized. Unlike off-shore wind generators, which can be damaged by storms or high seas, tidal or maritime flow windmills run just beneath the water surface or are firmly affixed to the seabed. Most buried tidal turbines are similar to wind turbines in that they are attached to the ocean bottom and are propelled by water rather than wind. These turbine have a vertical rotation and work similarly to a regular mill, with a rotor, gear, and electricity generator. These three components are attached to a steel support system, which can be a gravity framework, a sunk piling framework, or a squat framework, as indicated.

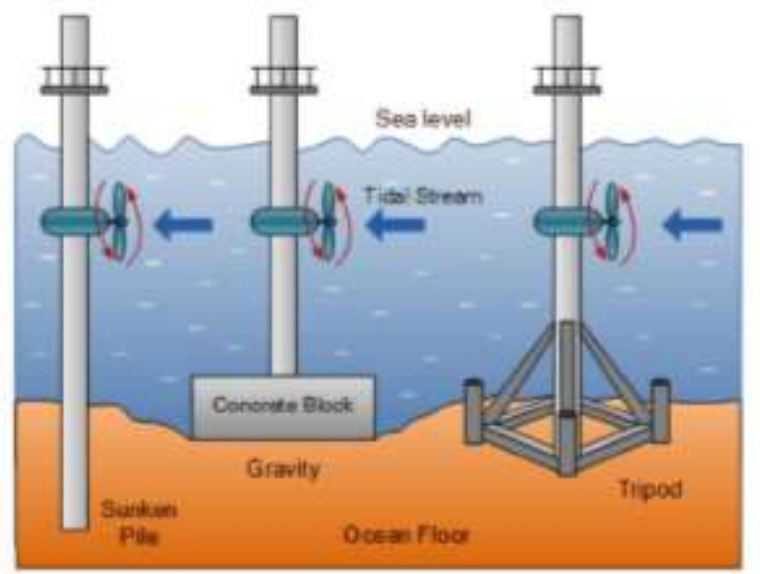

Figure 1 Design for Tidal Stream Generator

The installation of turbines in tidal streams is complicated since the devices are big and disturb the tide that they are attempting to capture. Depending on the size of the turbine and the location of the tidal stream, the environmental effect might be devastating. In shallow water, turbines are most efficient. This generates more energy and enables ships to navigate around the turbines. The turbine blades of a tidal generator also slowly turn which helps marine creatures from being entangled in the system.

\section{B. Barrages}

A large dam known as a barrage is used in another form of tidal energy generator. Because the dam is low, water can pour over the top or through turbines in the dam with a barrage. Barrages can be built over tidal rivers, bays, and estuaries (the wide part (mouth) of a river where it joins the sea). Turbines inside the barrage capture the force of the tides in the same manner as a river dam does. As the sea rises, the barrage gates open. The barrage gates close at high tide, producing a lake or tidal lagoon. The water is subsequently discharged through the barrage's turbines, which generate electricity at a rate that engineers can regulate.

If necessary, barricades can be created to elevate the level of water at the entrance sufficiently to extract the needed flow. Bombardments and weirs are commonly provided with controlling gates to keep downstream levels of water as low as possible during high flows and storms. These gates can be controlled to keep the appropriate water level. The longitudinal wave, also known as a tidal power plant, is a form of "marine renewable energy" system that uses lengthy separators, dams, sluice gate entrances, or tidal locks to collect and store the sea's energy stored. A longitudinal wave is a type of tidal energy technology scheme that involves the construction of a true low-walled dam, sometimes known as a "tidal barrage." The barrage's base is built at sea level, with the doors barely just above water's surface. There are a number of passageways below the water's surface that 
regulate the stream of fluid thru the turbine generator power. Sluices are the name for these tunnels.

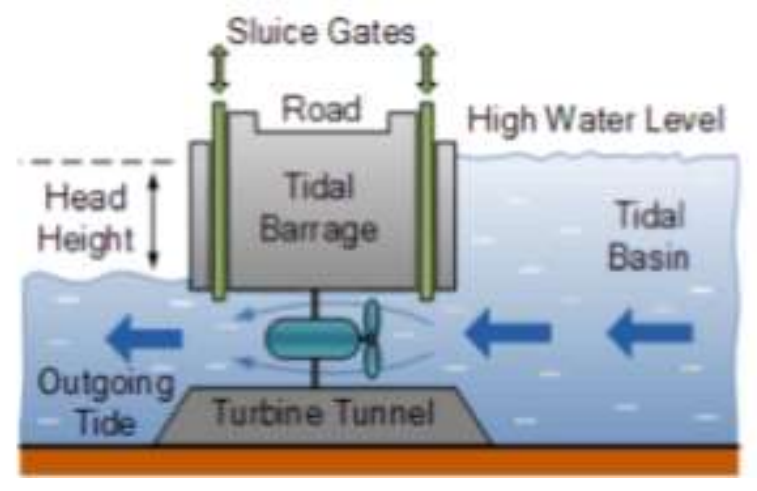

Figure 2 Tidal Barrage Flood Generation system

The water that flows in and out of sluices has a tremendous quantity of kinetic energy, which is recovered as much as possible by this mechanism and then transformed to power. The only distinction between this technique of energy extraction and hydropower is that with a tidal barrage, water flows in two directions rather than one. Entering tides, or high tides, fill the reservoir with water, but departing tides, or low tides, flow in the reverse way and empty it. Because tide is the horizontal flow of water due to gravity force exerted by the Sun and Moon, this approach takes use of this natural phenomenon and produces the most significant kind of fuel, electric.

\section{Tidal Lagoon}

A tidal lagoon is a body of ocean water partially surrounded by a natural or man-made barrier. Freshwater empties into tidal lagoons, which are also known as estuaries. A tidal energy producer that uses tidal lagoons would work similarly to a barrage. However, unlike barrages, tidal lagoons may be built along the natural coastline. Continuous energy might be generated by a tidal lagoon power plant. As the lagoon fills and empties, the turbines spin.

A added to the water is a power plant that uses the evident \& fall of sea levels to make electricity. Tidal lagoons, like tidal barrages, capture a significant volume of water behind such a man-made barrier before releasing it to spin turbines. A tidal lagoon encloses a section of shoreline with a large tidal range behind such a barrier, with a layout meticulously tailored for the local environment, unlike a barrage, which spans a main river delta in a single direction environment.

Tidal lagoons have a little environmental impact. Natural resources such as rock can be used to build the lagoons. At low tide, they would show as a low breakwater (sea wall), and at high tide, they would be submerged. Smaller creatures could swim inside the structure, and animals could swim around it. Large predators such as sharks would be unable to enter the lagoon, allowing smaller fish to thrive. Birds would most likely swarm the area. However, the energy production from tidal lagoon generators is likely to be low.

The waters in the lagoons then recovers to a temperature that is nearly identical to that of the sea outdoors. So because rotors are 'bi-directional,' power can be generated from both input and output waves, this process occurs in reversal as the tidal rushes out (ebbs).

\section{LITERATURE REVIEW}

Pankaj Warak et al. [1] One of the most significant forms of aquatic energy is tide energy. Wave energy is derived from the ups and downs of the ocean's water level, which naturally occurs. The maximum and minimum tides are created when the angle formed by the sun and the moon with respect to the earth equals zero degrees, and are known as spring tides. Neap tides occur when the moon and sun form a 90-degree angle with respect to the earth. The resulting tides are not quite as high or low as they are at other periods of the month. Due to the non, pure, dependable, and quasi character, tidal power is among the most predictable and viable forms of wave and tidal. To capture tidal energy, a variety of approaches such as tidal stream and tidal current rotors are being developed. As a result, they offer the best chance of harvesting energy from the waters. The location of Indian floods and certain tide locations around the world, as well as tidal energy plants around the world, are described in this study.

Dr. Sajjad Zaidi et al. [2] Several tidal turbine concepts were examined in this research, as well as their application by different countries. Afghanistan is now experiencing a 300MW electricity shortage. Demand will continue to rise, necessitating the implementation of more initiatives and projects. Pakistan's growing energy demands necessitate prioritising energy choices based on a variety of characteristics for alternative energy generation, including renewables, generation costs, and the lifetime of the different sources accessible in Pakistan. Pakistan's coastline, which stretches for around 1040 kilometres, can be effectively utilised to address the rising need for power, particularly in coastal regions and localities.

Youcef Belkhier et al. and Abdelyazid Achour et al. [3] This work focuses on the development of a novel apathy management for a PMSG-based tidal power converter. The models and methods for the PMSG passivity-based voltage regulator have been presented. The simulated results, including the kinematics of the PMSG for a certain tidal speed, demonstrate the efficacy of the power converter. Simulation tests show that the highest tidal power point can be tracked quickly, and the reactance provided by the suggested technology is quite low. The PMSG system has a strong efficiency, a lot of resilience against generating 
parametric uncertainty, and it's also quite efficient. The control approach is a realistic and reasonably simple architecture that has a lot of potential for tidal power uses. The modeled factors led is now being prepared for experimental verification.

P. Suwanapingkarl [4] The authors sincerely recognise the extraordinary assistance given by the Electrical Generation Administration of Thailand (EGAT) for the financial, collaborative technical information, and integrated development effort of the 'E8-PowerBuoys' device under contract no. 61-S101000-11- SS03S3008356. The authors gratefully acknowledge the technological data provided by the Rajamangala Universiti teknologi Phra Nakhon (RMUTP), Bangkok, Thailand, King Mongkut's Universiti teknologi Thonburi (KMUTT), Bangkok, Thailand, and the College of Northumbria at Norwich (UNN), Newcastle, U.k.. The authors would like to express their gratitude to the members of the $3 \mathrm{GreeN}$ by $\mathrm{KP}$ research team for their wonderful assistance and collaboration.

Y. Zhang et al. [5] Modeling smaller, relevant devices or combining CFD with various prediction approaches has been advocated by researchers. Nonetheless, turbulent modelling has been a critical subject in CFD approaches, and a lot of time and effort has gone into studying turbulent and wave effects on the wake and turbine operation. The current research provides a review of the effects of CFD on the efficiency of tidal current turbines in both steady and transient flows. The actuation and blades resolution approaches correctly predicted the turbine's efficiency, which was confirmed in the lab. The wake's findings, interpretations of the blades force, liquid technique and onset flow models, and upstream range length, have been both similar and discordant with observations. In the case of arrays, initial research indicates that turbine layout can have a significant impact on the onset flow and, as a result, the efficiency of nearby turbine rows. The data, such as wake Stochastic speed decreases and changing turbine power in unstable flows, tend to validate the wind resemblance hypothesis. Due to the flow's convection velocity, the wake rebounds quicker than in a steady flow state under the effect on the surface waves, although the mean results remained nearly similar.

M.B.R. Topper et al. [6] A theoretical model of the link between levelized cost of energy cost of energy (LCOE) and mean mechanically yearly power generation (MMAEP) is built to test the validity of these claims for small, region arrays. The rate of energy lost to interactions as TECs are added to an ideally placed array determines the model's behaviour into three classes; however, only one class has the highest MMAEP correlating to the lowest LCOE. A modern optimization algorithm is integrated to the sophisticated ocean energy electronica simulation tool 'DTOcean' and applied to arrays of TECs confined by a 2 ha base station to test this concept. The DTOcean results are demonstrated to be accurately described by the hypothetical LCOE model up to and including 12 TECs deployed. The level of TEC interaction typically increases when 13 TECs are deployed, contradicting the hypothetical model. However, enabling bad experiences between TECs reduces the LCOE dramatically, lowering it by $47.8 \%$ compared to the best quasi array.

G.M. Jónsdóttir and F. Milano [7] The periodic variations of the Irish having similar to wind and/or tidal generation are discussed in this study for several scenarios. Except for when waves are included in the tidal stream, simulation results demonstrate that tidal production does not contribute larger frequency changes than wind. In this situation, the tidal generation's output fluctuates on a regular basis, causing the system's speed to fluctuate significantly. The research also demonstrates how periodic variations in tidal barrages can be reduced by incorporating adequate frequency control.

D. Hu et al. [8] This research offers a general EMPC paradigm for power management in marine current turbines. In comparison to the typical pitch control system method, the suggested control scheme can limit standby generator to a safe level and greatly reduce pitch movement, hence improving system efficiency. The power at the source side is efficiently regulated by the generation control system, which decreases the gear loss of the gearbox, lowering the tower fatigue load and reducing gear chatter. Even though the collected energy power is lowered when compared to traditional control methods, the full power generation system's service life is assured. The system's total economic advantage has increased.

C. Lafleur et al. [9] This study presents a methodology for assessing the financial potential of integrating tidal power in a distant community, which can be utilised by community or developers as a quick screening tool. HOMER Pro's features are used to model the life - cycle costs of tidal projects. The local price of diesel fuel (as a metric of the cost of electricity), the electricity load scale, and the local tidal current resource are used to describe towns. In order to find a straightforward relationship between the economic feasibility and these variables and tide rotor power rating combos, a series of HOMER Pro simulations were run. Three parameters evolved as a broad structure for determining the economic viability of adding tidal power in a rural town.

Mingxing Wen et al. [11] Tidal energy is a renewable energy source that is both clean and environmentally favourable. It has a number of advantages, including long-term viability, minimal startup costs, ample supply, and excellent predictability due to its regular pattern. The tidal power converter (TECS) uses the angular momentum of ocean 
waves induced by heavenly gravitation to power a turbine that is connected to a generator to produce electricity. However, due to physical architecture of the generating, the output power is significantly non-linear and is closely related to water velocity, orientation, load quantity, and so on. The moon phase, which can be regarded a predictable component, has a strong influence on the liquid speed and direction of the tidal stream. However, quasi forces such as the sea are present. The peak power (MPP) must be continuously monitored and recorded. The ideal tip speed ratio (TSR) approach, the optimal relationship based (ORB) technique, and the perturb and observe $(\mathrm{P} \& \mathrm{O})$ method are all examples of state-of-the-art MPP tracking (MPPT) of TECS in this work. We summarise the concepts, benefits, and limits of the examined approaches, and demonstrate the effectiveness of MPPT based on P\&O in TECS using simulation results.

Advantages and disadvantages of Tidal Energy

\section{a. Advantages}

1. Being a renewable and sustainable energy source, tidal energy reduces the dependence on fossil fuels.

2. No liquid or solid pollutants are produced.

3. The energy from tides can be stored for future use.

4. In contrast to wind energy, tidal currents are both predictable and reliable.

5. Tidally driven coastal currents generate an energy density which is four times greater than air.

\section{b. Disadvantages}

1. The Tidal energy sources cannot be easily transported for long distances.

2. Energy generation can get disrupted by adverse weather conditions.

3. Tidal power can disrupt the habitats of aquatic life such as fishes, water mammals etc.

4. It is only appropriate for towns that are close to a tidal body of water.

5. The cost to set up the tidal power plants is exceedingly large.

\section{Electricity generation potential}

Since many tidal energy methods are not commercially viable, wave power contributes just a small percentage of world energy now. Nevertheless, because flood waters include a lot of useful power, there is a lot of potential for it to be used. The amount of energy stored in tides is 3,000 gigawatts ( $\mathrm{GW}$; billion watts), however estimations about how much of that power is accessible for tidal barrage electricity production range from 120 to $400 \mathrm{GW}$, depending on site and converting capability. In comparison, an average modern coal-fired power station generates approximately 550 megawatts (MW; million watts). Despite the fact that overall worldwide electricity usage reached 21,000 terawatt-hours in 2016 (one terawatt $[\mathrm{TW}]=$ one trillion watts), energy experts believe that completely built-out tidal power systems will reduce total global energy usage.

A few of these technology was becoming available on the market by the early twenty-first century. The Subsystem Lake Tidal Energy Plant in South Korea, which generates $254 \mathrm{Mw}$ of power, is the world's largest tidal power station. Since the 1960s, a 240 MW tidal lagoon nuclear plant in La Rance, France, has been in operation, with a normal output of 0.5 terawatt-hour annually. Larger energy production initiatives are on the way; for example, in August 2017, the first stage of the MeyGen initiative in Scotland's Inner Sounds produced 700 megawatt-hours of electricity. The tidal lagoon systems, which can disturb estuarine habitats throughout construction and maintenance, have sparked environmental issues regarding tidal powerplants. Turbines and tide barriers are planned.

\section{CONCLUSION}

Tidal current can be used in power generation with exact predictability and continuity regardless of seasons. Even the high tidal current speed would yield large energy generation, not many locations possess these advantages. This paper focusses on the tidal energy and the utilization of this energy in generation of power. It has many advantages such as sustainability, low initial cost, abundant and highly predictable due to its regular pattern. Numerous tidal turbine technologies were discussed along with their implementation by various countries. In addition to this we have also discussed the drawbacks of the

\section{REFERENCE}

[1] P. Warak and P. Goswami (2020). An explanation of how tidal energy is used to produce energy.

[2] Suwanapingkarl, P., \& Srivallop, K. (2020). Reviews Existing Technologies and Proposes ' E8PowerBuoys ' Nano-Scale Generator Of Tidal And Wave Energy For River And Ocean. Icpei, 20192022.

[3] Abbas, M. K. (2021). Renewable Tidal Power Generation Significance \& Challenges. 12-16.

[4] Belkhier, Y., \& Achour, A. (2021). Passivity-based Voltage Controller for Tidal Energy Conversion System with Permanent Magnet Synchronous Generator. 19(X), 1-11. 
[5] Zhang, Y., Fernandez-rodriguez, E., Zheng, J., Zheng, Y., Zhang, J., Gu, H., Zang, W. E. I., \& Lin, X. (2020). A Review on Numerical Development of Tidal Stream Turbine Performance and Wake Prediction. $\quad 8, \quad 79325-79337$. https://doi.org/10.1109/ACCESS.2020.2989344

[6] Topper, M. B. R., Olson, S. S., \& Roberts, J. D. (2021). On the benefits of negative hydrodynamic interactions in small tidal energy arrays. Applied Energy, 297(January), 117091. https://doi.org/10.1016/j.apenergy.2021.117091

[7] Jónsdóttir, G. M., \& Milano, F. (2020). Stochastic Modeling of Tidal Generation for Transient Stability Analysis: A Case Study based on the All-Island Irish Transmission System is. Electric Power Systems Research, 189(August), 106673. https://doi.org/10.1016/j.epsr.2020.106673

[8] Hu, D., Teng, Y., \& Wu, F. (2020). Optimal Control Design of Generator Systems for Marine Current Turbine Applications Using Economic Model Predictive Control. 8. https://doi.org/10.1109/ACCESS.2020.3038472

[9] La, C., Al, W., Cousineau, J., Hiles, C. E., Buckham, B., \& Crawford, C. (2020). A screening method to quantify the economic viability of off-grid in- stream tidal energy deployment. 159, 610-622. https://doi.org/10.1016/j.renene.2020.05.102

[10] Mingxing Wen; N. Srikanth "Maximum power point tracking for tidal turbine generator", 2017 Asian Conference on Energy, Power and Transportation Electrification (ACEPT), IEEE, DOI: 10.1109/ACEPT.2017.8168579.

[11]Ahmed G. Abo-Khalil \& Ali S. Alghamdi, 2021. "MPPT of Permanent Magnet Synchronous Generator in Tidal Energy Systems Using Support Vector Regression," Sustainability, MDPI, Open Access Journal, vol. 13(4), pages 1-15, February. 